\title{
CERN-INO magical Beta-beam experiment: A high precision probe for neutrino parameters
}

\author{
Sanjib Kumar Agarwalla*t \\ Department of Physics, Virginia Tech, \\ Blacksburg, VA 24061, USA \\ E-mail: sanjibevt.edu
}

\section{Sandhya Choubey}

Harish-Chandra Research Institute,

Chhatnag Road, Jhunsi, Allahabad 211019, India

E-mail: sandhya@hri.res.in

\section{Amitava Raychaudhuri}

Harish-Chandra Research Institute,

Chhatnag Road, Jhunsi, Allahabad 211019, India

E-mail: raychaudehri.res.in

This talk is an attempt to underscore in detail the physics reach of an experimental set-up where neutrinos produced in a beta-beam facility at CERN would be observed in the proposed large magnetized iron calorimeter detector (ICAL) at the India-based Neutrino Observatory (INO). The "magical" CERN-INO beta-beam set-up offers an excellent avenue to use the "Golden" channel $\left(v_{e} \rightarrow v_{\mu}\right)$ oscillation probability for a simultaneous determination of the neutrino mass ordering and $\theta_{13}$ avoiding the impact of the CP phase $\delta_{C P}$ on these measurements. With Lorentz boost $\gamma=650$ and irrespective of the true value of $\delta_{C P}$, the neutrino mass hierarchy could be determined at $3 \sigma$ C.L. if $\sin ^{2} 2 \theta_{13}$ (true) $>5.6 \times 10^{-4}$ and we can expect an unambiguous signal for $\theta_{13}$ at $3 \sigma$ C.L. if $\sin ^{2} 2 \theta_{13}$ (true) $>5.1 \times 10^{-4}$ independent of the true neutrino mass hierarchy.

10th International Workshop on Neutrino Factories, Super beams and Beta beams

June 30 - July 52008

Valencia, Spain

\footnotetext{
*Speaker.

$\dagger$ It is my pleasure to acknowledge the support of Harish-Chandra Research Institute (HRI) where the work has been done. The computational work presented in this talk has been performed using the HRI cluster facilities. I would like to thank Anselmo Cervera for providing me the local hospitality during Nufact08.
} 


\section{Introduction}

Future long baseline neutrino oscillation experiments will play a crucial role to measure the third mixing angle $\theta_{13}$, the $\operatorname{sign}^{1}$ of $\Delta m_{31}^{2} \equiv m_{3}^{2}-m_{1}^{2}\left(\operatorname{sgn}\left(\Delta m_{31}^{2}\right)\right)$ and the CP phase $\left(\delta_{C P}\right)$ using the $v_{e} \rightarrow v_{\mu}$ transition probability $\left(P_{e \mu}\right)$, often referred to in the literature as the "golden channel" [1]. An absolutely pure and intense $v_{e}$ (or $\bar{v}_{e}$ ) flux can be produced using "beta-beams" [2] at CERN and oscillations can be observed through muons produced via $v_{\mu}$ (or $\bar{v}_{\mu}$ ) at ICAL@INO [3]. Interestingly, the CERN-INO distance of $7152 \mathrm{~km}$ happens to be tantalizingly close to the so-called "magic baseline" $[4,5]$ where the sensitivity to the neutrino mass ordering $\left(\operatorname{sgn}\left(\Delta m_{31}^{2}\right)\right)$ and more importantly, $\theta_{13}$, goes up significantly, while the sensitivity to the unknown CP phase is absent. This permits such an experiment involving the golden $P_{e \mu}$ channel to make precise measurements of the mixing angle $\theta_{13}$ and neutrino mass hierarchy avoiding the issues of intrinsic degeneracies [6] and correlations [1] which plague other baselines. This large baseline also captures a nearmaximal matter-induced contribution to the oscillation probability. In this talk, we will discuss in detail the physics prospects of this CERN-INO magical beta-beam project.

\section{2. “Golden channel” oscillations}

The expression for $P_{e \mu}$ in matter, upto second order terms in the small quantities $\alpha \equiv \Delta m_{21}^{2} / \Delta m_{31}^{2}$ and $\theta_{13}$, is given by $[1,7]$ :

$$
\begin{aligned}
P_{e \mu} & \simeq \sin ^{2} 2 \theta_{13} \sin ^{2} \theta_{23} \frac{\sin ^{2}[(1-\hat{A}) \Delta]}{(1-\hat{A})^{2}} \pm \alpha \sin 2 \theta_{13} \xi \sin \delta_{C P} \sin (\Delta) \frac{\sin (\hat{A} \Delta)}{\hat{A}} \frac{\sin [(1-\hat{A}) \Delta]}{(1-\hat{A})} \\
& +\alpha \sin 2 \theta_{13} \xi \cos \delta_{C P} \cos (\Delta) \frac{\sin (\hat{A} \Delta)}{\hat{A}} \frac{\sin [(1-\hat{A}) \Delta]}{(1-\hat{A})}+\alpha^{2} \cos ^{2} \theta_{23} \sin ^{2} 2 \theta_{12} \frac{\sin ^{2}(\hat{A} \Delta)}{\hat{A}^{2}}
\end{aligned}
$$

where $\Delta \equiv \Delta m_{31}^{2} L /(4 E), \xi \equiv \cos \theta_{13} \sin 2 \theta_{12} \sin 2 \theta_{23}$, and $\hat{A} \equiv \pm\left(2 \sqrt{2} G_{F} N_{e} E\right) / \Delta m_{31}^{2} . G_{F}$ and $N_{e}$ are the Fermi coupling constant and the electron density in matter, respectively. The second term of Eq. 2.1 is positive (negative) for neutrinos (antineutrinos). The sign of $\hat{A}$ is positive (negative) for neutrinos (antineutrinos) with $\mathrm{NH}$ and it is opposite for $\mathrm{IH}$.

\section{The CERN-INO magical Beta-beam set-up}

A particularly interesting scenario arises when $\sin (\hat{A} \Delta)=0$, the last three terms in Eq. 2.1 drop out and the $\delta_{C P}$ dependence disappears from the $P_{e \mu}$ channel which provides a clean ground for the determination of $\theta_{13}$ and $\operatorname{sgn}\left(\Delta m_{31}^{2}\right)$. Since $\hat{A} \Delta= \pm\left(2 \sqrt{2} G_{F} N_{e} L\right) / 4$ by definition, the first non-trivial solution for $\sin (\hat{A} \Delta)=0$ reduces to $\rho L=\sqrt{2} \pi / G_{F} Y_{e}$, where $Y_{e}$ is the electron fraction inside the Earth. This gives $\frac{\rho}{[\mathrm{g} / \mathrm{cc}]} \frac{L}{[\mathrm{~km}]} \simeq 32725$, which for the PREM [8] density profile of the earth is satisfied for the "magic baseline", $L_{\text {magic }} \simeq 7690 \mathrm{~km}$. The CERN-INO distance corresponds to $L=7152 \mathrm{~km}$, which is pretty close to this magic baseline.

This large baseline requires traversal through denser regions of the earth. Thus, for neutrinos (antineutrinos) with energies in the range 3-8 GeV sizable matter effects are induced for $\mathrm{NH}(\mathrm{IH})$. A unique aspect of this set-up is the possibility of observing near-resonant matter effects in the $P_{e \mu}$ channel. In fact, for this baseline, the average Earth matter density calculated using the PREM

\footnotetext{
${ }^{1}$ The neutrino mass hierarchy is termed "normal (NH)" ("inverted (IH)") if $\Delta m_{31}^{2}=m_{3}^{2}-m_{1}^{2}$ is positive (negative).
} 

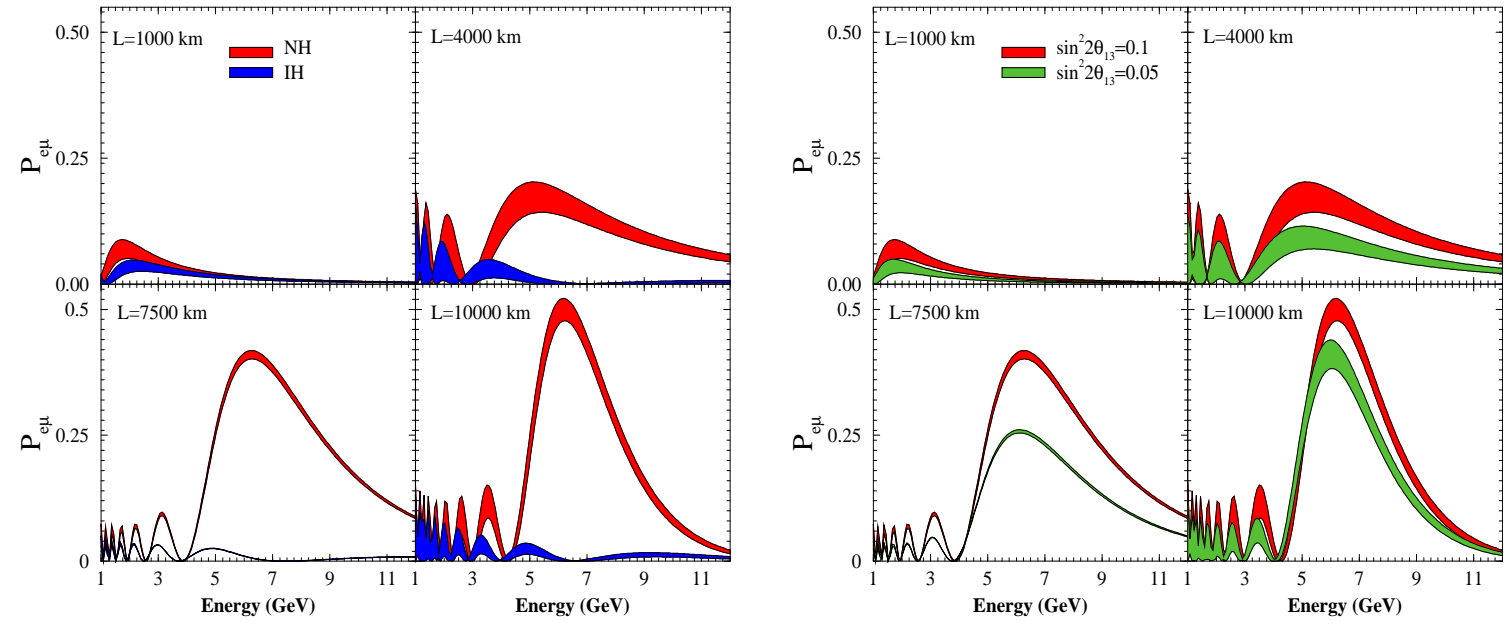

Figure 1: Both the panels show the energy dependence of $P_{e \mu}$ for four baselines where the band reflects the effect of the unknown $\delta_{C P}$. Left panel depicts the effect of $\delta_{C P}$ in making distinction between NH \& IH with $\sin ^{2} 2 \theta_{13}=0.1$. Right panel reflects the difference in $P_{e \mu}$ for two different values of $\sin ^{2} 2 \theta_{13}$ with NH.
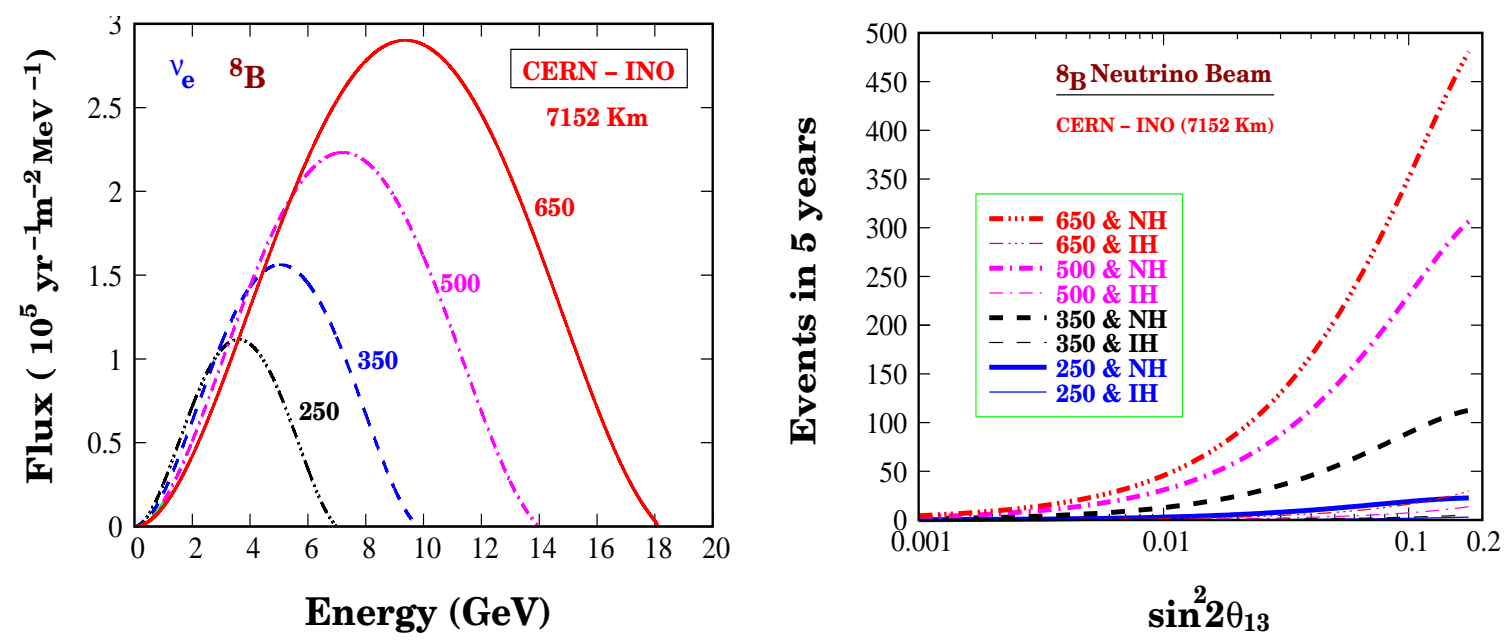

Figure 2: Left panel shows the boosted unoscillated spectrum of neutrinos from ${ }^{8} \mathrm{~B}$ ion which will hit the ICAL@INO detector, for four different benchmark values of $\gamma$. Right panel depicts the expected $\mu^{-}$events in 5 years as a function of $\sin ^{2} 2 \theta_{13}$ with a total detector mass of $50 \mathrm{kton}$ and $80 \%$ detection efficiency. The value of $\gamma$ and the hierarchy chosen corresponding to each curve is shown in the figure legend.

profile is $\rho_{a v}=4.17 \mathrm{~g} / \mathrm{cc}$, for which the resonance energy is $E_{\text {res }} \equiv\left|\Delta m_{31}^{2}\right| \cos 2 \theta_{13} / 2 \sqrt{2} G_{F} N_{e}=$ $7.45 \mathrm{GeV}$, taking $\left|\Delta m_{31}^{2}\right|=2.5 \times 10^{-3} \mathrm{eV}^{2}$ and $\sin ^{2} 2 \theta_{13}=0.1$.

We present all our results assuming certain benchmark values [9] for the oscillation parameters $\left|\Delta m_{31}^{2}\right|=2.5 \times 10^{-3} \mathrm{eV}^{2}, \sin ^{2} 2 \theta_{23}=1.0, \Delta m_{21}^{2}=8.0 \times 10^{-5} \mathrm{eV}^{2}, \sin ^{2} \theta_{12}=0.31$ and $\delta_{C P}=0$. The exact neutrino transition probability $P_{e \mu}$ using the PREM density profile is given in Fig. 1. For neutrinos (antineutrinos), matter effects in long baselines cause a significant enhancement in $P_{e \mu}$ for $\mathrm{NH}(\mathrm{IH})$, while for $\mathrm{IH}(\mathrm{NH})$, the probability is almost unaffected. This feature can be used to determine the neutrino mass hierarchy (see left panel of Fig. 1). For $L=7500 \mathrm{~km}$, which is close to the magic baseline, the effect of the CP phase is seen to be almost negligible. This allows a clean 

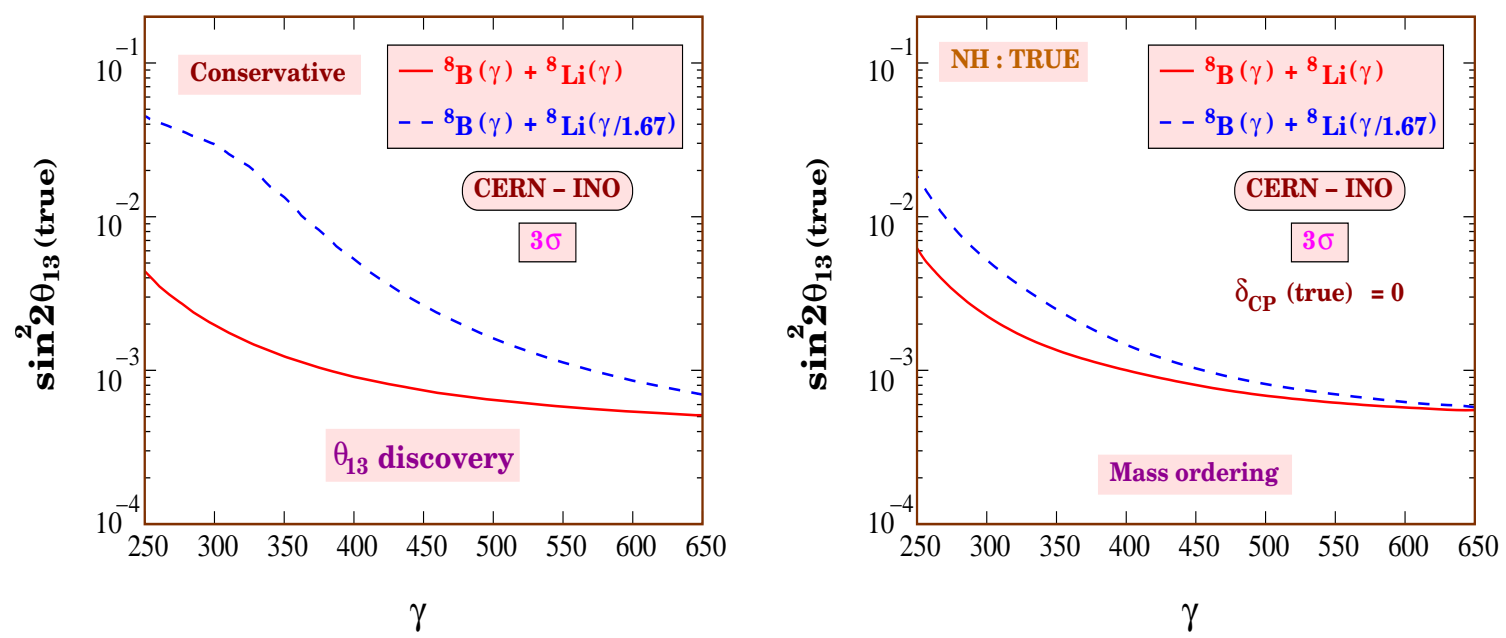

Figure 3: Left panel shows the $3 \sigma$ discovery reach for $\sin ^{2} 2 \theta_{13}$ (true). Right panel shows the minimum value of $\sin ^{2} 2 \theta_{13}$ (true) for which the wrong inverted hierarchy can be ruled out at the $3 \sigma$ C.L., as a function of the Lorentz boost $\gamma$. The red solid lines in both the panels are obtained when the $\gamma$ is assumed to be the same for both the neutrino and the antineutrino beams. The blue dashed lines show the corresponding limits when the $\gamma$ for the ${ }^{8} \mathrm{Li}$ is scaled down by a factor of 1.67 with respect to the $\gamma$ of the neutrino beam, which is plotted in the $x$-axis.

measurement of $\operatorname{sgn}\left(\Delta m_{31}^{2}\right)$ (see left panel of Fig. 1) and $\theta_{13}$ (see right panel of Fig. 1), while for all other cases the impact of $\delta_{C P}$ on $P_{e \mu}$ is appreciable.

Zucchelli [2] put forward the novel idea of a beta-beam [10, 11, 12, 13, 14, 15, 16, 17, 18, 19], which is based on the concept of creating a pure, well understood, intense, collimated beam of $v_{e}$ or $\bar{v}_{e}$ through the beta-decay of completely ionized radioactive ions. We consider ${ }^{8} \mathrm{~B}\left({ }^{8} \mathrm{Li}\right)$ ion [20] as a possible source for a $v_{e}\left(\bar{v}_{e}\right)$ beta-beam. The end point energies of ${ }^{8} \mathrm{~B}$ and ${ }^{8} \mathrm{Li}$ are $\sim 13-14$ $\mathrm{MeV}$. For the Lorentz boost factor $\gamma=250(500)$ the ${ }^{8} \mathrm{~B}$ and ${ }^{8} \mathrm{Li}$ sources have peak energy around $\sim 4(7) \mathrm{GeV}$. We can see from Fig. 2 (left panel) that with $\gamma=500$, the $v_{e}$ spectrum peaks nearby $E_{\text {res }}$. We assume that it is possible to get $2.9 \times 10^{18}$ useful decays per year for ${ }^{8} \mathrm{Li}$ and $1.1 \times 10^{18}$ for ${ }^{8} \mathrm{~B}$ for all values of $\gamma$.

The ICAL@INO detector, capable of detecting muons along with their charge, is planned to have a total mass of $50 \mathrm{kton}$ at startup, which might be later upgraded to $100 \mathrm{kton}$. The INO facility is expected to come up at PUSHEP (lat. North $11.5^{\circ}$, long. East $76.6^{\circ}$ ), situated close to Bangalore in southern India. This constitutes a baseline of $7152 \mathrm{~km}$ from CERN. The detector will be made of magnetized iron slabs with interleaved active detector elements. For ICAL, glass resistive plate chambers have been chosen as the active elements. The expected number of events are shown in the right panel of Fig. 2. We take a detector with an energy threshold of $1 \mathrm{GeV}$, detection efficiency of $80 \%$ and charge identification efficiency of $95 \%$.

The $\theta_{13}$ sensitivity reach is defined as the range of $\sin ^{2} 2 \theta_{13}$ which is incompatible with the data generated for $\sin ^{2} 2 \theta_{13}$ (true) $=0$ at the $3 \sigma$ C.L. This performance indicator corresponds to the new $\sin ^{2} 2 \theta_{13}$ limit if the experiment does not see a signal for $\theta_{13}$-driven oscillations. At $3 \sigma$, the CERNINO beta-beam set-up can constrain $\sin ^{2} 2 \theta_{13}<1.14 \times 10^{-3}$ with five years of running of the betabeam in both polarities with the same $\gamma=650$ and full spectral information. The $\theta_{13}$ discovery 
reach is defined as the range of $\sin ^{2} 2 \theta_{13}$ (true) values which allow us to rule out $\sin ^{2} 2 \theta_{13}=0$ at the $3 \sigma$ C.L. We present our results in the left panel of Fig. 3, as a function of $\gamma$. The plot presented show the most conservative numbers which have been obtained by considering all values of $\delta_{C P}$ (true) and both hierarchies. The mass hierarchy sensitivity is defined as the range of $\sin ^{2} 2 \theta_{13}$ (true) for which the wrong hierarchy can be excluded at the $3 \sigma$ C.L. The results are depicted as a function of $\gamma$ in the right panel of Fig. 3. For NH true, the $\operatorname{sgn}\left(\Delta m_{31}^{2}\right)$ reach corresponds to $\sin ^{2} 2 \theta_{13}$ (true) $>$ $5.51 \times 10^{-4}$, with 5 years energy binned data of both polarities and $\gamma=650$. Here we had assumed $\delta_{C P}($ true $)=0$. However, as discussed before, the effect of $\delta_{C P}$ is minimal due to the near magic baseline and hence we expect this sensitivity to be almost independent of $\delta_{C P}($ true).

\section{Conclusions}

In this talk, we discussed the expected physics performance of the CERN-INO magical betabeam set-up. The results are very impressive, comparable to those expected in neutrino factories.

\section{References}

[1] A. Cervera et al., Nucl. Phys. B 579, 17 (2000) [Erratum-ibid. B 593, 731 (2001)].

[2] P. Zucchelli, Phys. Lett. B 532, 166 (2002); C. Volpe, J. Phys. G 34, R1 (2007).

[3] See http://www.imsc.res.in/ ino.

[4] V. Barger, D. Marfatia and K. Whisnant, Phys. Rev. D 65, 073023 (2002).

[5] P. Huber and W. Winter, Phys. Rev. D 68, 037301 (2003); A. Y. Smirnov, arXiv:hep-ph/0610198.

[6] J. Burguet-Castell et al., Nucl. Phys. B 608, 301 (2001); H. Minakata and H. Nunokawa, JHEP 0110, 001 (2001); G. L. Fogli and E. Lisi, Phys. Rev. D 54, 3667 (1996).

[7] M. Freund, P. Huber and M. Lindner, Nucl. Phys. B 615, 331 (2001).

[8] A. M. Dziewonski and D. L. Anderson, Phys.Earth Planet.Interiors 25, 297 (1981).

[9] M. Maltoni et al., New J. Phys. 6, 122 (2004), arXiv:hep-ph/0405172 v6.

[10] P. Huber et al., Phys. Rev. D 73, 053002 (2006); J. Burguet-Castell et al., Nucl. Phys. B 725, 306 (2005); J. Burguet-Castell et al., ibid. 695, 217 (2004); J. E. Campagne et al., JHEP 0704, 003 (2007); A. Donini et al., Phys. Lett. B 641, 432 (2006); P. Coloma et al., JHEP 0805, 050 (2008).

[11] S. K. Agarwalla, A. Raychaudhuri and A. Samanta, Phys. Lett. B 629, 33 (2005).

[12] S. K. Agarwalla, S. Choubey and A. Raychaudhuri, Nucl. Phys. B 771, 1 (2007).

[13] S. K. Agarwalla, S. Choubey and A. Raychaudhuri, Nucl. Phys. B 798, 124 (2008).

[14] R. Adhikari, S. K. Agarwalla and A. Raychaudhuri, Phys. Lett. B 642, 111 (2006).

[15] S. K. Agarwalla, S. Rakshit and A. Raychaudhuri, Phys. Lett. B 647, 380 (2007).

[16] S. K. Agarwalla, S. Choubey, S. Goswami and A. Raychaudhuri, Phys. Rev. D 75, 097302 (2007).

[17] S. K. Agarwalla, S. Choubey, A. Raychaudhuri and W. Winter, JHEP 0806, 090 (2008).

[18] S. K. Agarwalla, S. Choubey and A. Raychaudhuri, Nucl. Phys. B 805, 305 (2008).

[19] W. Winter, arXiv:0804.4000 [hep-ph]; W. Winter, arXiv:0809.3890 [hep-ph].

[20] C. Rubbia et al., Nucl. Instrum. Meth. A 568, 475 (2006); C. Rubbia, arXiv:hep-ph/0609235. 